\title{
Dolutegravir-Based ART: Exploring Patient Safety and Acceptability Following National Roll-Out in Uganda
}

Henry Zakumumpa ( $\square$ zakumumpa.henry09@gmail.com )

Makerere University College of Health Sciences

Freddy Eric Kitutu

Makerere University

Helen Bomire Ndagije

National Drug Authority

Nakitto-Kesi Diana

National Drug Authority

Jacquellyn Nambi Ssanyu

Makerere University

Ronald Kiguba

Makerere University College of Health Sciences

Research Article

Keywords: ART, patient, DTG, public

Posted Date: July 14th, 2021

DOl: https://doi.org/10.21203/rs.3.rs-670284/v1

License: (c) (i) This work is licensed under a Creative Commons Attribution 4.0 International License.

Read Full License 


\section{Abstract}

\section{BACKGROUND}

The World Health Organization recommends dolutegravir (DTG) as the backbone for first-line and secondline antiretroviral therapy (ART) worldwide. However, little is known about the acceptability and tolerability of DTG-based ART at routine points-of-care in Uganda. We set out to explore the perceptions of ART clinic managers regarding the acceptability and tolerability of DTG-based ART since national roll-out in March 2018 in Uganda.

\section{METHODS}

We adopted a qualitative descriptive design involving 49 ART clinic managers and clinicians. Between September 2020 and February 2021, we conducted 22 in-depth interviews with ART clinic managers and clinicians in 12 purposively selected health facilities across Uganda. The selection of study sites ensured diversity in facility ownership-type (public/private), level of service delivery (tertiary/secondary/primary) and the four major geographic sub-regions of Uganda. We conducted three focus group discussions with 27 ART clinicians in the participating facilities. Data were analyzed by thematic approach.

\section{RESULTS}

The participants acknowledged that DTG-based ART is well tolerated by the majority of their patients who appreciate the reduced pill burden, perceived less side effects and superior viral load suppression. However, they reported that a number of patients experience adverse drug reactions (ADRs) after being transitioned to DTG. Hyperglycemia is, by far, the most commonly reported suspected ADR associated with DTG-based regimens and was cited in all but two participating facilities. Insomnia, weight gain and reduced libido are among the other frequently cited suspected ADRs. In addition, ART clinic managers perceived some of the suspected ADRs as resulting from drug interactions between dolutegravir and isoniazid. Weak diagnostic capacities and shortage of associated commodities (e.g. glucometers and test kits) were reported as impediments to understanding the full extent of ADRs associated DTG-based ART.

\section{CONCLUSION}

While DTG-based regimens were perceived to be well tolerated by the majority of patients at participating facilities, a number of patients were reported to experience suspected ADRs key among which were hyperglycemia, insomnia and reduced libido. We recommend interventions aimed at strengthening blood glucose monitoring for patients taking DTG-based regimens and precautionary assessment of all patients prior to initiating them on DTG for known risk factors for DTG related ADRs.

\section{Background}


Universal access to anti-retroviral therapy (ART) is enshrined in the Sustainable Development Goals (SDG 3.3) and in UNAIDS's 95-95-95 targets for ending AIDS as a public health threat by 2030 [1]. Globally, there are 37.9 million people living with HIV [1]. Out of these, 24.5 million are currently accessing ART [1].

Following evidence from clinical trials that demonstrated that DTG-based ART had superior patient outcomes compared to alternative combinations (such as those containing efavirenz) [2], [3], the World Health Organization (WHO) revised and published guidelines recommending dolutegravir (DTG)-based ART as the first-line regimen in HIV treatment [2], [3].

In addition, there are studies suggesting that DTG may result in cost savings in procurements of antiretrovirals especially in countries in sub-Saharan Africa which are heavily dependent on international assistance for their national HIV responses [4], [5].

In Uganda, DTG-based ART was rolled-out nationally as the recommended first-line regimen starting in March 2018 following this WHO guidance [2], [3]. President's Emergency Plan for AIDS Relief (PEPFAR) the predominant HIV donor in Uganda [6] included DTG roll-out in its annual program targets for the national HIV response in Uganda [7]. Following PEPFAR programmatic guidance, procurement of ART commodities in Uganda was aligned accordingly.

In 2019, an aggressive 100-day roll-out of anti-tuberculosis medication known as isoniazid preventive therapy (IPT) was implemented across Uganda [10]. Tuberculosis is the leading cause of death of people living with HIV (PLHIV) [8]. Tuberculosis preventive therapy has been shown to reduce mortality in PLHIV [8].

Against the backdrop of the introduction of DTG-based ART as the new recommended first-line regimen [2] and the aggressive roll-out of IPT across Uganda [10], patient and medication safety concerns may have been overlooked [9]. The ambitious roll-out of these HIV care and treatment strategies were not accompanied by commensurate investments in robust pharmacovigilance systems. There is a dearth of empirical data exploring medication-related harms despite growing patient complaints of the safety profile of these newer HIV medications [11]. This development is in spite of the fact that anti-retroviral medicines contribute the highest number of case reports of adverse drug reactions (ADRs) of any medication in Uganda [9]. DTG-based ART is associated with an increase in the Body Mass Index of users (or becoming obese/ overweight) [14] and has come under the spot light following safety signals of neural tube defects in infants born to women exposed to DTG in the first three months of conception [15]. ART related ADRs are associated with morbidity and mortality in patients [16] and the economic burden of treating ADRs [17]. Hence, detecting medication harms early is critical to informing interventions for promoting patient safety.

Despite the critical importance of preventing medication harm, there is little research documenting the safety profile of newer HIV medications in Uganda following national roll-out of DTG-based ART in 2018. Most of the evidence base on the safety profile of DTG has been generated from clinical trials [2]- [4]. The 
safety profile of DTG in 'real-life', routine points-of-care in countries with a high HIV burden such as Uganda is not adequately understood [18]- [20].

We set out to explore the perceptions of ART clinic managers in Uganda regarding the acceptability and tolerability of DTG-based ART following national roll-out in March 2018.

\section{Methods}

\section{Study Design}

A qualitative descriptive research design was adopted [21]. We sought to understand the perspectives of frontline health workers regarding the safety and tolerability of DTG-based ART based on their occupational experiences of attending to recipients of HIV care at routine points-of-care in Uganda.

The roll out of DTG-based ART across Uganda since 2018 offered us a conducive two-year postimplementation evaluation platform for the present study [11]- [13].

\section{Study sites and sample selection}

We aimed for a diverse sample of health workers representing multiple contextual settings [22] with regard to the different levels of service delivery in the Ugandan health-system (tertiary I/secondary/primary), as shown in Fig. 1, as well as across a range of health worker cadres (medical doctor/clinical officer/nurse etc.) [22] and varied geographical sub-regions of Uganda (Northern, Eastern, Western and Central) [23]. To this end, a purposive sample of health facilities was selected. Table 1 shows that we selected four Regional Referral Hospitals (RRHs) which were geographically representative of the four regions of Uganda. In each of the four regions of Uganda, we selected three health centres at the secondary level of care (Health Centre IVs) and primary care level (Health Centre IIIs). 
Table 1

Characteristics of case-study facilities and participants

\begin{tabular}{|llll|}
\hline Health facility & Level of care & $\begin{array}{l}\text { Geographic sub- } \\
\text { region }\end{array}$ & $\begin{array}{l}\text { Number of } \\
\text { participants }\end{array}$ \\
\hline 1.Jinja RRH & $\begin{array}{l}\text { Regional Referral } \\
\text { Hospital }\end{array}$ & Central & 3 \\
\hline 2.Mbale RRH & $\begin{array}{l}\text { Regional Referral } \\
\text { Hospital }\end{array}$ & Eastern & 3 \\
\hline 3.Lira RRH & $\begin{array}{l}\text { Regional Referral } \\
\text { Hospital }\end{array}$ & Northern & 3 \\
\hline 4. Mbarara RRH & $\begin{array}{l}\text { Regional Referral } \\
\text { Hospital }\end{array}$ & Western & 7 \\
\hline 5.Bugembe HC IV (Jinja) & $\begin{array}{l}\text { Health Centre IV } \\
\text { Health Centre IV }\end{array}$ & Central & 4 \\
\hline 6.Namatala HC IV (Mbale) & Eastern & 3 \\
\hline 7.Ogur HC IV (Lira) & Health Centre IV & Northern & 6 \\
\hline $\begin{array}{l}\text { 8.Bwizibwera HC IV } \\
\text { (Mbarara) }\end{array}$ & Health Centre IV & Western & 2 \\
\hline 9.Kakira HC III (Jinja) & Health Centre III & Eastern & 4 \\
\hline 10.Maluku HC III ( Mbale) & Health Centre III & Eastern & 4 \\
\hline $\begin{array}{l}\text { 11.Amuca HC III (PNFP) } \\
\text { Lira }\end{array}$ & Health Centre III & Northern & 6 \\
\hline $\begin{array}{l}\text { 12.Rubindi HC III } \\
\text { (Mbarara) }\end{array}$ & Health Centre III & Western & 49 \\
\hline & Total & & 4 \\
\hline
\end{tabular}

We envisaged that this purposive sampling of health facilities would generate a diverse cadre of health workers (e.g. medical doctors, clinical officers, nurses) who ordinarily attend to recipients of HIV care [24] and potentially encounter ADRs in the course of their routine practice or regular health services delivery. We selected at least three health workers from each of the 12 health facilities (Table 1 ). The unit of participant selection was the HIV clinic at each of the 12 participating facilities.

\section{Data collection}

Between September 2020 and February 2021, we conducted a total of 22 In-depth interviews (IDIs) with ART clinic managers and clinicians at the participating facilities to elicit their perspectives on patient safety and the acceptability of DTG-based ART among recipients of HIV care and treatment. We particularly sought to interrogate patient experiences of taking DTG-based ART as reported to attending health workers at the HIV clinics at participating facilities [25], [26]. 
In addition, we conducted three focus group discussions (27 participants) with clinicians at the HIV clinics of participating facilities who have managed patients taking DTG-based regimens for at least 12 months. A focus group discussion (FGD) guide was constructed for use during the proceedings of the FGDs. The aim of the FGDs was to elicit the collective experiences of clinicians as a group [27] regarding the perceived safety and tolerability of DTG-based ART as reported to them by patients attending their HIV clinics. We purposively selected clinicians at participating facilities who were in service at the onset of transitioning patients to DTG-based ART and could offer retrospective insights on patient safety. The focus groups were conducted by the first author who has an academic background in the social sciences and extensive experience in qualitative health services research [28]-[30]. The first author was assisted by two research assistants who took notes during the proceedings and operated the recorder.

Furthermore, we conducted eight Key Informant Interviews (KIIs) with national-level HIV program managers seeking to understand national-level dynamics underpinning [32] the transitioning of patients to DTG-based ART such as donor programmatic targets.

\section{Data Analysis}

We followed the processes recommended for ensuring rigour in case-study and qualitative data analysis suggested by Miles \& Huberman (1990) [33]. We made audio recordings of all of the interviews and then transcribed each interview verbatim. In terms of data analysis procedures, we followed four major steps. However, this was a largely iterative process [34]. The first step involved data familiarization through multiple readings of the interview transcripts by $\mathrm{HZ}, \mathrm{JN}$ and $\mathrm{DN}$. The second step entailed generating a coding framework. Codes were inductively generated from the interview transcripts by $\mathrm{HZ}$ and $\mathrm{DN}$. The developed coding scheme was applied to the interview and focus group transcripts. We utilized the same coding scheme to explore themes and sub-themes for both the interviews and focus group discussion. The third stage was that of abstracting the coded data into thematic categories. The emergent inductive or data-driven codes were then grouped under thematic matrices [35]. The fourth and final step was that of Interpretation and overall synthesis [36] which involved all the authors (HZ, FK, HN, DN, JN, RK).

\section{Results}

The results emerging from this qualitative study are presented in three sections. Section A reports the demographic characteristics of study participants. Section B reports on the perceived advantages of DTG-based ART. Section C documents suspected ADRs associated with DTG-based ART.

\section{SECTION A: Participants by health worker cadre}

In terms of health worker cadre, Clinical Officers $(n=15)$ were the most represented in our study. They were followed by nurses $(n=10)$ and medical doctors $(n=6)$. Participants included 'expert patients' or lay workers which is not uncommon at HIV clinics across Uganda [22].

Table 2 shows the health worker cadres that participated in the study $(n=49)$. 
Table 2

Cadre of health workers who participated in the study

\begin{tabular}{|ll|}
\hline Cadre of health worker & $\mathbf{n}$ \\
\hline 1.Medical doctors & 6 \\
\hline 2. Clinical Officers & 15 \\
\hline 3.Nursing cadre & 10 \\
\hline 4.Pharmacy cadre & 4 \\
\hline 5.Midwife & 2 \\
\hline 6.Data analysts & 2 \\
\hline 7.Laboratory technicians & 2 \\
\hline 8.Lay workers ('expert patients') & 8 \\
\hline & 49 \\
\hline
\end{tabular}

It is common to find that lay health workers are engaged in clinical HIV disease management at HIV clinics across Uganda. Lay workers are a very diverse, informal group of health workers typically with a minimum of secondary school formal education who are co-opted on the staff of HIV clinics in order to fill critical staffing shortages [24].

In terms of gender, $51 \%$ of participating health workers were male while $49 \%$ were female.

\section{SECTION B: Advantages of DTG-based ART over alternative regimens:}

There was consensus among health workers (HWs) that DTG-based regimens were well tolerated by the majority of their patients. When asked to estimate the percentage of patients who report DTG-associated ADRs, the most frequently mentioned percentage range was between $5 \%$ and $10 \%$.

'The majority of our patients are doing well on DTG and have no complaints. Actually, most of the patients prefer DTG. There are some who are unsatisfied with it (DTG). Some have mild side effects which they are tolerating. Many of these patients have been on drugs (ART) for some time. They have learnt to tolerate them (side effects)' [Medical doctor, Regional Referral Hospital, Western Uganda]

However, several HWs were quick to add that the numbers of patients experiencing suspected DTGassociated ADRs were still a concern for them as service providers. 
HWs identified several advantages of DTG-based over alternative regimens key among which was the widely-held perception that it had a better viral suppression rate.

Patients were reported to have expressed several advantages of DTG which included a less pill burden, having less side effects and improved portability of medication packages (due to less bill burden).

\section{Better viral load suppression}

HWs frequently mentioned that DTG had a superior viral suppression rate when compared with other regimens especially efavirenz-based ART. When probed on why they perceived this to be the case, HWs pointed out several cases of patients whose viral load has not been adequately suppressed while on nonDTG regimens but were subsequently virally suppressed after being transitioned to DTG.

We had many patients in our clinic who were non-suppressors but became (viral) suppressors when we put them on TLD (DTG). In my experience of treating patients, TLD is better at viral load suppression [Clinical Officer, Health Centre IV, Eastern Uganda]

\section{Less side effects}

Health workers reported that DTG actually had less side effects when compared with alternative regimens. It was frequently mentioned that patients experienced less side effects compared to efavirenzbased ART. The side effects that patients frequently mentioned as having diminished after switching from efavirenz-based regimens include dizziness, headaches and nightmares.

'Actually most of the patients prefer DTG because of having less side effects. You know most of them here are farmers. So once they take it in the morning, they can even go to their gardens to till the land without feeling drowsy or the dizziness. Some like to take it in the morning because even if they don't take food, at least they can go and dig in the morning and come back and eat so it has been good'. [Medical doctor, Regional Referral Hospital, Northern Uganda].

\section{Less pill burden}

Patients were reported to be relieved at the reduced bill burden after being transitioned to DTG-based regimens. Although recipients of HIV care were previously required to swallow multiple tablets of antiretrovirals, with the introduction of DTG, they were only required to swallow a single tablet once-daily. One of the clinicians we talked to who was also living with HIV indicated that even the size of the DTG tablet was a lot smaller in size compared to earlier ones.

'The pill burden has reduced. With DTG you only need to take one tablet a day. In years past, the volume of pills one had to take were intimidating. You literally swallowed pills of all colours. But with DTG it is just a single pill every day' [Clinical Officer, Health Centre IV, Eastern Uganda].

\section{Improved ARV packaging}


One of the advantages of DTG-based regimens described by patients to health workers was the improved portability of medication packaging. The once-daily, single tablet translated into a reduced load of medication to be carried home by patients. In addition, it was reported that the available medication package for DTG provided to patients in Uganda required less plastic bottles compared to alternative regimen packaging.

'With this new (DTG) packaging of a three-month supply in one tin they are happy. Someone has to carry only one tin in their pockets for (medication lasting) three months and you just go!. Initially, if they were to dispense to you for six months, it meant that six tins. Now they are just two tins, for six months and you really feel, yeah!' [Health worker, Health Centre III, Western Uganda].

It emerged that the reduced weight and bulk of (DTG) medication packaging helped reduce the risk of HIV-related stigma because medication packages were less conspicuous. Patients appreciated the newer packaging as it reduced potential curiosity in the community of what load they carried in their bags. Furthermore, the reduced medication package bulk helped in instances where couples had not yet disclosed their HIV status to their long-term partners. Patients who struggle with HIV sero-status disclosure were reported to have said that it is easier to conceal medication packages from their partners which helped in their adherence to ART.

\section{SECTION C: Most commonly cited suspected adverse reactions}

Although HWs reported that DTG is well tolerated by the majority of patients, there were a number of patients who report suspected ADRs.

\section{Dolutegravir-induced hyperglycemia}

Hyperglycemia was by far the most commonly cited DTG-associated ADR across our case-study facilities. Hyperglycemia was reported as a DTG-associated ADR in 10 (out of the 12) participating facilities. In our sample of facilities, the largest number of patients reporting DTG-associated ADRs was at the tertiary level of care. Table 2 shows the number of suspected DTG-associated ADRs at four Regional Referral Hospitals. However, complaints of hyperglycemia were common across case study facilities. Hyperglycemia stood out prominently among DTG-associated ADRs.

'The main issue with DTG is hyperglycemia. Some of my patients have been admitted in the ward with very high blood sugars. Today I have just removed a middle-aged woman from DTG and taken her back to TLE (efavirenz). They had a blood sugar reading of 20. [Medical doctor, Health Centre IV, Eastern Uganda].

\section{Gaps in baseline blood sugar screening}

The majority of case-study facilities, including at the tertiary-level of care, reported that during the initial roll out of DTG which commenced in 2018 , they did not conduct baseline blood glucose tests. This was 
attributed to the initial low awareness, among HWs, around the association between DTG and hyperglycemia. The shortage of glucometers and accompanying test kits in case study facilities was frequently cited as a fundamental barrier to blood glucose monitoring for patients taking DTG-based ART.

'There was no baseline or screening of blood sugars before transitioning patients. There were resource constraints. You need glucometers, test kits and all those kind of things and the IP (PEPFAR implementing partner) did not have a budget for that. So we are transitioning patients to DTG without baseline screening for diabetes' [Medical doctor, Regional Referral Hospital, Northern Uganda].

Due to commodity shortages, health workers indicated they frequently advise patients to make private out-of-pocket expenditure on blood glucose tests to rule out new-onset hyperglycemia. However, patients were reportedly often unable to raise the 5,000 shillings (US \$1.23) charged for tests by private providers.

Participating facilities reported being given programmatic targets for transitioning the majority of their patients to DTG-based ART by PEPFAR implementing organizations in their geographic sub-regions. In the case of the four participating Regional Referral Hospitals (3 out 4) had transitioned over $80 \%$ of their HIV client loads to DTG-based regimens. Lower-level facilities (particularly Health Centre IIIs) had a much lower rate of transition to DTG regimens (on average $30 \%$ ). It emerged from HW discourses that although the programmatic targets set for them for transitioning patients to the more efficacious DTG-based regimens were understandable, there was insufficient attention paid to patient safety in roll-out targets.

\section{Deaths due to suspected dolutegravir-induced hyperglycemia}

The gap in blood glucose monitoring for patients on DTG was suspected to have resulted in the deaths of three patients at two participating primary care facilities. Health workers indicated that in two of the fatalities, they discovered belatedly that the patients had undiagnosed underlying diabetic conditions after they had already been transitioned to DTG. The absence of a policy on compulsory blood glucose screening during the initial phase of DTG roll-out in 2018 and the shortage of associated commodities was suspected to have contributed to the escalation in the diabetic conditions of patients especially those that had been undiagnosed.

\section{Increased appetite}

Increased appetite after being initiated on DTG was cited by multiple HWs as a common complaint among patients. The medication was said to induce abnormal appetites in some patients during the course of DTG-based therapy.

'We have had clients who complain about overeating. They have come and told me when they take the drug (DTG) the demand for food becomes abnormal. They tell me, "With this drug (DTG) you need to eat and eat. You eat several times". In fact, for some patients when you want to transition them to DTG they tell you, "my friends tell me you need to have a lot of food, how am I going to manage?". [Nurse, Health Centre IV, Eastern Uganda]. 
Weight gain among patients was another frequently cited side effect by ART clinic managers. Although unexpected weight gain was reported directly by patients without prompting, HWs reported observing weight gain based on trends in weight readings among some patients who had been taking DTG after multiple weeks.

\section{Insomnia}

Insomnia was one of the most commonly reported suspected ADR associated with DTG-based regimens by participants. ART clinic managers reported that patients complained of an inability to sleep at night after taking DTG. These reports were fairly consistent across case-study facilities.

'Lack of sleep at night is a common complaint among patients. Some people are affected and some are not affected. [Medical doctor, Regional Referral hospital, Northern Uganda].

It emerged from our interviews with ART clinic managers that complaints of insomnia were partly attributable to patients' non-adherence to medication instructions. Although attending HWs advised patients to take DTG-based medication during morning hours, many of the patients were actually taking it at night as was the previous practice with efavirenz-based ART. This emerged as a potential contributory factor in the multiple cases of insomnia across case-study facilities.

'Sometimes patients do not take our instructions seriously. You tell them to take this medicine in the morning hours and instead they take it at night. Later on they get those problems of insomnia and the others that is when they come and tell you 'musawo' (doctor) I have not been sleeping at night. Then you ask them when have you been taking this medicine? That's when you learn they have been taking it at night' [Pharmacist, Health Centre HC IV, Western Uganda].

Hence, patient behavior and practices in taking DTG-based regimens appeared to be of interest. For instance, it emerged that some patients preferred to take DTG-based regimen at night instead of the mornings due to HIV-related stigma at their workplaces.

'HIV-related Stigma is it part of the problem. They fear to take ARVs (antiretrovirals) during the day when people are seeing them say in the offices or markets where they work' [Nurse, Health Centre III, Eastern Uganda].

The majority of case-study districts are predominantly rural and many of the patients derive their upkeep from agrarian livelihoods. Patients complained that when they take DTG in the morning hours it weakens them physically yet they earn their living from tilling the land.

'Patients tell us that from their experience, when they take the medicine in the morning they feel they don't get enough (physical) energy to do their normal businesses. So they would prefer to take it after supper at night. They will say 'I have a busy routine every morning. I would have to go to the kitchen first to prepare food every morning in order to swallow the medicine. I don't have time'. [ Clinical Officer, Health Centre IV, Eastern Uganda]. 
Health workers reported that due to early-morning occupational routines, many of the patients, contrary to instructions, take DTG at night for the first handful of days. It is only when patients experience insomnia they revert to taking DTG in the morning hours as advised.

\section{Reduced libido in adult males}

Several adult males reportedly complain of reduced libido within a few weeks of being transitioned to DTG-based regimens. Health workers reported that they are approached by a number of adult males who complain of a low sex drive and a noticeable decline in interest in sex after transitioning to DTG. Health workers recounted tales of male patients' complaints from their regular female sexual partners of sexual dysfunction. Requests to revert to efavirenz-based regimens by male patients experiencing a low sex drive were cited across multiple case-study facilities.

'The main problem that I have seen is loss of libido for men they say 'omulilo gugenze' (the fire has gone). Since I was put on DTG, I don't feel like having sex. This new drug is affecting me'. It is true there is loss of libido. And you wonder whether to take the patient back to the former regimen and versus the recommended one but loss of libido has been there' [Nurse, Regional Referral Hospital, Eastern Uganda].

Participants reported that the prevalence of reduced libido post-transition to DTG may be higher than is actually reported to HWs because several males do not open up due to fear of embarrassment owing to cultural notions of masculinity [36].

With regard to male patients who actually report reduced sex drive, HWs reported that they frequently switch them back to their former regimens (more commonly efavirenz-based ART). Sex drive was said to improve in many cases after switching from DTG-based regimens.

"I have so far helped five males who developed low sex drive on transition to TLD, we changed them back to TLE(Tenofovir, Lamiduvine, Efavirenz) and now they are doing well" [ART clinic manager, Health Centre IV, Eastern Uganda].

\section{Suspected drug interactions between DTG and Isoniazid}

Health workers reported that there were possible drug interactions between DTG-based ART and isoniazid (INH) in the suspected ADRs reported by patients. It emerged that DTG was rolled out countrywide in 2018 and a year later, the anti-tuberculosis medication (INH)- was also rolled out nationally. Health workers were emphatic in relaying the notion that initiating patients on these two medicines concurrently could result in ADRs. At a case-study Regional Referral Hospital, 12 deaths were reported of patients initiated on both DTG and INH.

'We have had over twelve deaths when I go back to a year ago (July 2019). These were patients who were on TLD (dolutegravir) and INH (isoniazid). Some were emergencies referred to us from private providers and some coming from lower-level facilities. We would review them and find that they were on both of these drugs' [ART clinic manager, Regional Referral Hospital, Western Uganda]. 
Health workers however expressed extreme difficulty in disentangling ADRs associated with DTG from those attributable to isoniazid. They reported that whenever patients complained of suspected ADRs when they were newly initiated on both DTG and INH, they were hard pressed in making causal links due to multiple limitations including a paucity of robust data on patients.

'For the first two weeks they will come with complaints such as headache and many other issues. You find they are complaining of weakness and you reach somewhere you fail to understand which drug to stop or maintain. You ask yourself. Should I stop INH or should I stop TLD? And it becomes a paradox'.

[Clinical Officer, Health Centre IV, Western Uganda]

It emerged from our interviews that during the initial roll-out of DTG in 2018 and INH in 2019, concurrent introduction of both medications was permitted. However, subsequent guidance in the updated national ART treatment guidelines of 2020 advised against concurrent introduction of the two medications.

It emerged in this study that switching back to former regimens was a common strategy by HW whenever patients complained of suspected DTG-associated ADRs.

'There was a 55-year old female. I transitioned her from TLE (efavirenz) to TLD (dolutegravir). On the third day she called me with all sorts of complaints; dizziness, headaches, general body weakness, a sore throat, she could not pass a fluid. I counselled her, that it might be due to another cause let her take it for a week but in the second week she returned it she said 'No. it is too much' I discussed it with my pharmacy clinician. We put her back to her former TLE (efavirenz) and she did well so after a week we were like we needed to record it as an adverse drug reaction'. [Clinical Officer, Health Centre IV, Western Uganda]

Health workers reported that in many instances ADRs cease once patients are switched back to efavirenzbased ART. However, health workers complained of shortages of efavirenz or TLE regimens due to a donor emphasis on DTG-based regimens former treatment regimens.

'The challenge we have is that there is no efavirenz at our facility. When patients who react badly to DTG emerge, I start running here and there to get the drug. We begin running to nearby facilities to see if they have any. They are not stocking TLE (efavirenz-based ART) anymore so what will we do when we need efavirenz?' [Nurse, Health Centre IV, Eastern Uganda].

In our sample of health facilities, we observed that the higher the percentage of patients transitioned to DTG, the more the cases of suspected ADRs reported by HWs. For example, all but one of the Regional Referral Hospitals (RRH) in our sample had transitioned at least $80 \%$ of their patient populations to DTGbased regimens. RRHs also reported the highest cumulative number of suspected DTG-associated ADRs. Although this could be due to having significantly higher HIV client loads, the tertiary-level of care appeared to have noticeably higher cases of suspected ADRs associated with taking DTG. 


\section{Discussion}

Much of the literature on the safety profile of DTG-based ART has come from clinical trials. There is a dearth of evidence on the acceptability and tolerability of DTG-based ART in sub-Saharan African countries rolling out these newer HIV medications. We set out to explore the perceptions of ART clinic managers in Uganda regarding the acceptability and tolerability of DTG since initial roll-out in March 2018. While ART clinic managers acknowledged that DTG-based ART was well tolerated by the majority of their patients, they reported that a number of them suffer ADRs after being transitioned to the regimen. Hyperglycemia was the most commonly cited suspected ADR associated with DTG-based regimens. Insomnia, reduced libido and weight gain were among the other frequently cited suspected ADRs across case-study facilities. Furthermore, ART clinic managers perceived some ADRs as resulting from the concurrent use of DTG and isoniazid preventive therapy (IPT).

Dolutegravir-induced hyperglycemia was reported in all (but two) of the 12 participating facilities. The two facilities that didn't report hyperglycemia were lower-level facilities which had transitioned less than $30 \%$ of their HIV client loads to DTG-based regimes (compared to Regional Referral Hospitals which had transitioned over $80 \%$ of their patients to DTG). Our findings point to a critical need for blood glucose monitoring among patients taking DTG-based regimens [13], [37], [38]. ART clinic managers reported that at the onset of transitioning patients to DTG commencing in 2018, they did not carry out baseline blood glucose tests due to their initial lack of awareness around the association between DTG and hyperglycemia [13]. Even when this association became apparent, the shortage of commodities for testing blood glucose such as glucometers and test kits were indicated as fundamental constraints at participating facilities. Uganda is heavily dependent on external donors for HIV treatment with PEPFAR being the primary source of international assistance [28]- [31]. ART clinic managers reported that regionally-based PEPFAR implementing organizations were not budgeting for blood glucose monitoring in their HIV support programs. Furthermore, providers indicated that patients could not afford out-ofpocket expenditure on blood glucose tests at private outlets even when they were strongly recommended by attending clinicians. Whilst Uganda's national ART guidelines of 2020 were updated to include blood glucose monitoring for patients on DTG-based regimens, supply chain barriers continue to hamper observance of these guidelines [53]. Although previous studies have reported hyperglycemia as a DTGassociated ADR [37], [38], including a 2020 study in Uganda [13], our findings suggest that hyperglycemia could be more common than previously indicated. Further research utilizing more rigorous approaches and larger study samples is recommended. From the policy and programming lens, our study findings point to the need for increasing funding for blood glucose monitoring among HIV patients especially those on DTG-based regimens. In light of our findings, we add to the mounting calls for the integrated health services agenda especially around integrating HIV services with non-communicable diseases (NCDs) responses such as with regard to the management of diabetes mellitus and hypertension [40], [41].

Insomnia was the second most cited suspected DTG-associated ADR in our sample of facilities. Although it is unclear if this was independently associated with taking DTG, our study suggests that patient 
behavior is a contributory factor. More specifically, we found that it was common for patients to take DTG-based medication during night time contrary to explicit instructions from clinicians to take the tablet in the morning. A study by Nabitaka and colleagues [42] found that trouble sleeping was the third most prevalent self-reported side effect by patients who had been on DTG for at least six months. Previous studies have reported insomnia as an ADR associated with DTG [43]- [45], however our study shades more light on this phenomenon by suggesting a patient behavior dynamic. This calls for programming interventions aimed at improving adherence to medication instructions with regard to DTG-based regimens. Furthermore, our findings point to the need for increased sensitizations of patients prior to enrolling them to newer HIV medications [46]. For instance, patients were used to a routine of taking efavirenz-based regimens at night. However, DTG-based regimens demanded a radical shift to taking ART medication in the morning. Importantly, our study suggests a link between patients deliberately taking ART medication at night contrary to clinician instruction with HIV-related stigma or the fear by patients of taking this medication in the morning at the workplace in the presence of work colleagues [47]- [49].

An important finding of this study is that deficiencies in the laboratory infrastructure and commodity stock availability (such as for blood glucose test kits) at frontline points-of care are a barrier to determining the prevalence of suspected DTG-associated ADRs which could be higher than can be determined at the facility-level due to health-system constraints [50], [53]. For instance, ART clinic managers decried the lack of diagnostic tools and technologies for conducting regular liver function tests to identify suspected ADRs. This calls for increased investments in laboratory systems strengthening in Uganda to support early detection of suspected ADRs and prompt actions to secure patient safety for the over 1.2 million Ugandans enrolled on ART [28].

In this study, ART clinic managers especially at the tertiary level of care indicated that they were under pressure to meet donor targets of transitioning the majority of their HIV client loads to DTG-based regimens. Although donors' emphasis on transitioning to superior DTG-based regimens (such as in terms of viral load suppression) are understandable, it emerged that, from the perspective of providers, there has been insufficient attention paid to medication safety in the ambitious programmatic goals set for DTG transitions. In addition, HWs reported that they experienced shortage of efavirenz-based ART due to an on-going emphasis on transitioning to DTG-based regimens in Uganda. Health workers expressed concern that patients who experience DTG-associated ADRs and need to be switched to alternative regimens may not have these options available to them, which could severely affect their HIV treatment. Parkhurst and colleagues have observed the occasional tension between global actor interests and donor-recipient national interests [51].

Health workers suspected ADRs resulting from drug interactions between DTG and IPT. A hospital-based study by Griensven and colleagues [52] in Cambodia found that 'IPT discontinuation due to drug toxicity was common in patients subsequently initiating ART' highlighting the need for caution in initiating patients on both medications concurrently. The updated national ART treatment guidelines in Uganda of 2020 now advise against initiating patients on DTG and IPT concurrently. Wide dissemination and sensitizations of health workers on these updated national HIV treatment guidelines is critical in ensuring

Page 15/22 
patient safety from medication harm. The involvement of 'expert patients' in ART provision at case-study facilities underlines the importance of regular trainings in pharmacovigilance and in recognizing ADRs for all personnel in HIV clinics in Uganda. A study in Uganda reports that task shifting to non-clinician cadres is as high as $93 \%$ [24]. While health workforce shortages are undoubted, there is need for sensitizations on pharmacovigilance reporting for all who offer ART services in HIV clinics in Uganda which is listed by the WHO as one of the countries with a human resources for health crisis [21], [24].

\section{Limitations}

This study had multiple limitations which we wish to acknowledge. Our study utilized a sample of 12 health facilities. Case-study research designs by their very nature have inherent limitations in statistical generalizability of findings. However, our primary objective was to enable an in-depth examination of the safety profile of DTG-based at the frontline level of service delivery and from the perspective of health workers, given their operational contexts. In addition, it would perhaps have been more ideal to hear directly from the patients themselves or to learn from both health workers and patients taking DTG-based regimens. In-depth interviews and focus groups with patients are planned in a subsequent component of this study.

\section{Conclusion}

While DTG-based regimens were perceived to be well tolerated by the majority of patients at participating facilities, a number of patients were reported to experience suspected ADRs key among which were hyperglycemia, insomnia and reduced libido. We recommend interventions aimed at strengthening blood glucose monitoring for patients taking DTG-based regimens.

\section{List Of Abbreviations}

\section{AIDS}

Acquired Immune Deficiency Syndrome

\section{ADRs}

Adverse Drug Reactions.

ART

Anti-retroviral therapy

ARVs

Anti-retrovirals

DTG

Dolutegravir

\section{$\mathrm{MOH}$}

Ministry of Health

\section{PEPFAR}

The Presidents' Emergency Plan for AIDS Relief 
RA

Research Assistant

SSA

Sub-Saharan Africa

TLD

Tenofovir, Lamivudine, and Dolutegravir

TLE

Tenofovir, Lamivudine, and Efavirenz

WHO

World Health Organization

\section{Declarations}

Ethics approval and consent to participate:

Ethical approval was granted by Mildmay Uganda Research Ethics Committee(MUREC) under instrument: REC REF 0712-2020. MUREC is accredited by the Uganda National Council for Science and Technology (UNCST).

\section{Consent for publication}

Not applicable

\section{Availability of data and materials:}

The datasets generated during and/or analyzed during the current study are not publicly available due to ethical reasons but are available from the corresponding author on reasonable request.

\section{Competing interests:}

The authors declare that they have no competing interests.

\section{Funding}

This paper was written under the auspices of an early-career pharmacovigilance research grant funded by Uppsala Monitoring Centre (UMC) through the Consortium for Advanced Research Training in Africa(CARTA/2020/354.707A). Additional funding for data collection was received from the National Drug Authority (NDA) of Uganda through the Directorate of Product Safety.

\section{Authors' contributions:}

$\mathrm{HZ}$ conceptualized the study, collected the data, analyzed the data and produced the initial manuscript draft. FK, HN, DN, JN, RK contributed to data analysis and provided comments on the draft manuscript. All authors approved the final manuscript. 


\section{Acknowledgements}

We are very thankful to Julius Mayengo, Allan Sserwanga of the National Drug Authority of Uganda and Resty Nakayima (Research Assistant) for their support in field data collection across Uganda.

\section{References}

1. Bekker LG, Alleyne G, Baral S, Cepeda J, Daskalakis D, Dowdy D, Dybul M, Eholie S, Esom K, Garnett $G$, Grimsrud A. Advancing global health and strengthening the HIV response in the era of the Sustainable Development Goals: The International AIDS Society-Lancet Commission. The Lancet. 2018 Jul 28;392(10144):312 - 58.

2. WHO. Dolutegravir (DTG) and the fixed dose combination (FDC) of tenofovir/lamivudine/dolutegravir (TLD) Retrieved 1 Jun 2021 from: https://www.who.int/hiv/pub/arv/DTG-TLD-arv_briefing_2018.pdf

3. Phillips AN, Venter F, Havlir D, Pozniak A, Kuritzkes D, Wensing A, Lundgren JD, De Luca A, Pillay D, Mellors J, Cambiano V. Risks and benefits of dolutegravir-based antiretroviral drug regimens in subSaharan Africa: a modelling study. The Lancet HIV. 2019 Feb 1;6(2):e116-27.

4. Dorward J, Hamers RL. Dolutegravir in sub-Saharan Africa: context is crucial. The Lancet HIV. 2019 Feb 1;6(2):e72-3.

5. Zheng A, Kumarasamy N, Huang M, Paltiel AD, Mayer KH, Rewari BB, Walensky RP, Freedberg KA. The cost-effectiveness and budgetary impact of a dolutegravir-based regimen as first-line treatment of HIV infection in India. Journal of the International AIDS Society. 2018 Mar;21(3):e25085.

6. Zakumumpa H, Bennett S, Ssengooba F. Alternative financing mechanisms for ART programs in health facilities in Uganda: a mixed-methods approach. BMC health services research. 2017 Dec;17(1):1-1.

7. Uganda PEPFAR Country Operational Plan (COP) 2017. Retrieved 4 Nov 2019 from: https://www.state.gov/wp-content/uploads/2019/08/Uganda-18.pdf

8. Getahun H, Granich R, Sculier D, Gunneberg C, Blanc L, Nunn P, Raviglione M. Implementation of isoniazid preventive therapy for people living with HIV worldwide: barriers and solutions. Aids. 2010 Nov 1;24:S57-65.

9. National Drug Authority. National Pharmacovigilance Bulletin 2018. Retrieved 4 Feb 2020 from: https://www.nda.or.ug/nda/files/downloads/NDA-PVB-ISSUE-3-\%20FEB-2018-WEB(3).pdf

10. URC. Uganda launces 100-day plan to prevent TB among people living with HIV. Accessed 20 Feb 2020 from https://www.urc-chs.com/news/uganda-launches-100-day-plan-prevent-tb-among-peopleliving-hiv

11. Daily Monitor. People with HIV report side effects from new drug. Retrieved 9 Dec 2019 from: https://www.monitor.co.ug/News/National/People-with-HIV-report-side-effects-from-newdrug/688334-5345492-37f8xw/index.html

12. Laker EA, Arinaitwe A, Owarwo N, Onzia A, Nasasira B, Wailagala A, Kalule I, Anguzu G, Kiragga A, Seden K, Lwanga I. The potential teratogenicity alert for women conceiving on dolutegravir-based 
regimens: an assessment of risk communication by an urban HIV clinic in Uganda and choices made by women. Drug Safety. 2020 Nov;43(11):1133-40.

13. Lamorde M, Atwiine M, Owarwo NC, Ddungu A, Laker EO, Mubiru F, Kiragga A, Lwanga IB, Castelnuovo B. Dolutegravir-associated hyperglycaemia in patients with HIV. The Lancet HIV. 2020 Jul 1;7(7):e461-2.

14. Bourgi K, Rebeiro PF, Turner M, Castilho JL, Hulgan T, Raffanti SP, Koethe JR, Sterling TR. Greater weight gain in treatment-naive persons starting dolutegravir-based antiretroviral therapy. Clinical Infectious Diseases. 2020 Mar 17;70(7):1267-74.

15. Lyerly AD. Dolutegravir: advancing ethical research in pregnancy. The Lancet. 2019 Nov 30;394(10213):1972-4.

16. Keiser O, Fellay J, Opravil M, Hirsch HH, Hirschel B, Bernasconi E, Vernazza PL, Rickenbach M, Telenti A, Furrer H. Adverse events to antiretrovirals in the Swiss HIV Cohort Study: effect on mortality and treatment modification. Antiviral therapy. 2007 Jan 1;12(8):1157.

17. Dormann H, Muth-Selbach U, Krebs S, Criegee-Rieck M, Tegeder I, Schneider HT, Hahn EG, Levy M, Brune K, Geisslinger G. Incidence and costs of adverse drug reactions during hospitalisation. Drug safety. 2000 Feb;22(2):161-8.

18. Brehm TT, Franz M, Hüfner A, Hertling S, Schmiedel S, Degen O, Kreuels B, Zur Wiesch JS. Safety and efficacy of elvitegravir, dolutegravir, and raltegravir in a real-world cohort of treatment-naive andexperienced patients. Medicine. 2019 Aug;98(32).

19. Elzi L, Erb S, Furrer H, Cavassini M, Calmy A, Vernazza P, Günthard H, Bernasconi E, Battegay M. Adverse events of raltegravir and dolutegravir. AIDS (London, England). 2017 Aug 24;31(13):1853.

20. Mofenson LM, Pozniak AL, Wambui J, Raizes E, Ciaranello A, Clayden P, Ehrenkranz P, Fakoya A, Hill A, Khoo S, Mahaka I. Optimizing responses to drug safety signals in pregnancy: the example of dolutegravir and neural tube defects. Journal of the International AIDS Society. 2019 Jul;22(7):e25352.

21. Vaismoradi $\mathrm{M}$, Turunen $\mathrm{H}$, Bondas T. Content analysis and thematic analysis: Implications for conducting a qualitative descriptive study. Nursing \& health sciences. 2013 Sep;15(3):398-405.

22. Zakumumpa $\mathrm{H}$. Reimagining the role of the nursing workforce in Uganda after more than a decade of ART scale-up. Human Resources for Health. 2020 Dec;18:1-9.

23. Uganda Bureau of Statistics. Uganda Demographic and Health Survey. 2011.http://www.ubos.org/onlinefiles/uploads/ubos/UDHS/UDHS2011.pdf.

24. Zakumumpa H, Taiwo MO, Muganzi A, Ssengooba F. Human resources for health strategies adopted by providers in resource-limited settings to sustain long-term delivery of ART: a mixed-methods study from Uganda. Human resources for health. 2016 Dec;14(1):1-1.

25. Frank AW. The wounded storyteller: body, illness, and ethics. Chicago: University of Chicago Press; 2013.

26. Bury M. The sociology of chronic illness: a review of research and prospects. Sociol Health Illn. 1991;13:451-68. 
27. Seal DW, Bogart LM, Ehrhardt AA. Small group dynamics: The utility of focus group discussions as a research method. Group Dynamics: Theory, Research, and Practice. 1998 Dec;2(4):253.

28. Zakumumpa H, Rujumba J, Kwiringira J, Katureebe C, Spicer N. Understanding implementation barriers in the national scale-up of differentiated ART delivery in Uganda. BMC health services research. 2020 Dec;20(1):1-6.

29. Zakumumpa H, Paina L, Wilhelm J, Ssengooba F, Ssegujja E, Mukuru M, Bennett S. The impact of loss of PEPFAR support on HIV services at health facilities in low-burden districts in Uganda. BMC health services research. 2021 Dec;21(1):1-2.

30. Zakumumpa H, Rujumba J, Kwiringira J, Kiplagat J, Namulema E, Muganzi A. Understanding the persistence of vertical (stand-alone) HIV clinics in the health system in Uganda: a qualitative synthesis of patient and provider perspectives. BMC health services research. 2018 Dec;18(1):1-3.

31. Kakaire T, Schlech W, Coutinho A, Brough R, Parkes-Ratanshi R. The future of financing for HIV services in Uganda and the wider sub-Saharan Africa region: should we ask patients to contribute to the cost of their care?. BMC Public Health. 2016 Dec;16(1):1-5.

32. Gilson L, World Health Organization. Health policy and system research: a methodology reader: the abridged version. World Health Organization; 2013.

33. Miles MB, Huberman AM. Qualitative data analysis: An expanded sourcebook. sage; 1994 Jan 12.

34. Srivastava P, Hopwood N. A practical iterative framework for qualitative data analysis. International journal of qualitative methods. 2009 Mar;8(1):76-84.

35. Castleberry A, Nolen A. Thematic analysis of qualitative research data: Is it as easy as it sounds?. Currents in Pharmacy Teaching and Learning. 2018 Jun 1;10(6):807 - 15.

36. Jansz J. Masculine identity and restrictive emotionality. Gender and emotion: Social psychological perspectives. 2000 Mar 9:166-86

37. McLaughlin M, Walsh S, Galvin S. Dolutegravir-induced hyperglycaemia in a patient living with HIV. Journal of Antimicrobial Chemotherapy. 2018 Jan 1;73(1):258 - 60.

38. Odenyo JA. Prevalence of Dolutegravir Associated Hyperglycemia and Its Covariates Among Persons Living With HIV on Treatment at Kenyatta National Hospital (Doctoral dissertation, University of Nairobi). Retrieved 1 Jun 2021 from: http://erepository.uonbi.ac.ke/handle/11295/154331

39. Lo J, Oyee J, Crawford M, Grove R, DeMasi R, Curtis L, Fettiplace A, Vannappagari V, Payvandi N, Aboud M, van Wyk J. Dolutegravir and insulin resistance. CROI. 2019

40. Haldane V, Legido-Quigley H, Chuah FL, Sigfrid L, Murphy G, Ong SE, Cervero-Liceras F, Watt N, Balabanova D, Hogarth S, Maimaris W. Integrating cardiovascular diseases, hypertension, and diabetes with HIV services: a systematic review. AIDS care. 2018 Jan 2;30(1):103 - 15.

41. Duffy M, Ojikutu B, Andrian S, Sohng E, Minior T, Hirschhorn LR. Non-communicable diseases and HIV care and treatment: models of integrated service delivery. Tropical Medicine \& International Health. 2017 Aug;22(8):926-37. 
42. Nabitaka VM, Nawaggi P, Campbell J, Conroy J, Harwell J, Magambo K, Middlecote C, Caldwell B, Katureebe C, Namuwenge N, Atugonza R. High acceptability and viral suppression of patients on Dolutegravir-based first-line regimens in pilot sites in Uganda: A mixed-methods prospective cohort study. PloS one. 2020 May 27;15(5):e0232419.

43. Capetti AF, Di Giambenedetto S, Latini A, Sterrantino G, De Benedetto I, Cossu MV, Gori A. Morning dosing for dolutegravir-related insomnia and sleep disorders. HIV Med. 2018 May 1;19(5):e62-3.

44. Elliot ER, Wang X, Singh S, Simmons B, Vera JH, Miller RF, Fitzpatrick C, Moyle G, McClure M, Boffito $M$. Increased dolutegravir peak concentrations in people living with human immunodeficiency virus aged 60 and over, and analysis of sleep quality and cognition. Clinical Infectious Diseases. 2019 Jan 1;68(1):87-95.

45. Hoffmann C, Llibre JM. Neuropsychiatric adverse events with dolutegravir and other integrase strand transfer inhibitors. AIDS reviews. 2019 Jan 1;21(1):4-10.

46. Shoemaker SJ, de Oliveira DR, Alves M, Ekstrand $M$. The medication experience: preliminary evidence of its value for patient education and counseling on chronic medications. Patient education and counseling. 2011 Jun 1;83(3):443 - 50.

47. Rintamaki LS, Davis TC, Skripkauskas S, Bennett CL, Wolf MS. Social stigma concerns and HIV medication adherence. AIDS Patient Care \& STDs. 2006 May 1;20(5):359 - 68.

48. Katz IT, Ryu AE, Onuegbu AG, Psaros C, Weiser SD, Bangsberg DR, Tsai AC. Impact of HIV-related stigma on treatment adherence: systematic review and meta-synthesis. Journal of the International AIDS Society. 2013 Nov; 16:18640.

49. Camacho G, Kalichman S, Katner H. Anticipated HIV-related stigma and HIV treatment adherence: the indirect effect of medication concerns. AIDS and Behavior. 2020 Jan;24(1):185-91.

50. Ampadu HH, Hoekman J, Arhinful D, Amoama-Dapaah M, Leufkens HG, Dodoo AN. Organizational capacities of national pharmacovigilance centres in Africa: assessment of resource elements associated with successful and unsuccessful pharmacovigilance experiences. Globalization and health. 2018 Dec;14(1):1-7.

51. Parkhurst J, Ghilardi L, Webster J, Snow RW, Lynch CA. Competing interests, clashing ideas and institutionalizing influence: insights into the political economy of malaria control from seven African countries. Health policy and planning. $2021 \mathrm{Feb} ; 36(1): 35-44$.

52. van Griensven J, Choun K, Chim B, Thai S, Lorent N, Lynen L. Implementation of isoniazid preventive therapy in an HIV clinic in Cambodia: high rates of discontinuation when combined with antiretroviral therapy. Tropical Medicine \& International Health. 2015 Dec;20(12):1823-31.

53. Kagujje M, Mubiana ML, Mwamba E, Muyoyeta M. Implementation of isoniazid preventive therapy in people living with HIV in Zambia: challenges and lessons. BMC public health. 2019 Dec;19(1):1-4.

\section{Figures}




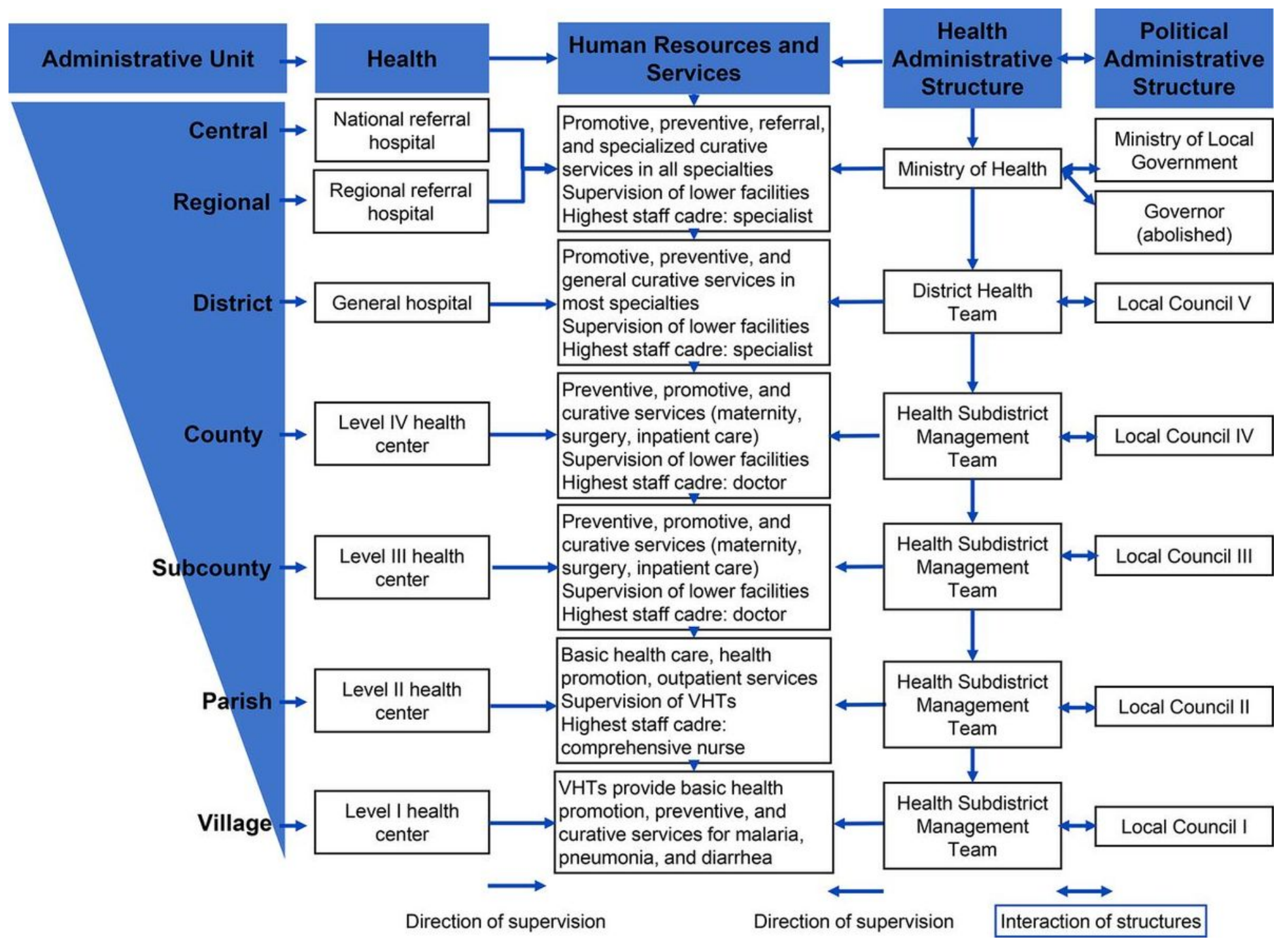

Figure 1

Uganda's Health and Administrative System Structure. Agnes Nanyonjo et al. Glob Health Sci Pract 2020;8:190-204 